\title{
5 Open Source GIS Geospatial Software for Archaeology: Towards its Integration into Everyday Archaeological Practice
}

\subsection{Introduction}

Geospatial software refers to all software employed to display, manipulate, analyse and produce geospatial information. Although this term is normally used for software classified as geographic information systems (GIS) it also includes others that can work with and produce geographically referenced information such as CAD, Remote Sensing or photogrammetric software. This paper focuses on GIS but references will be also made to other types of geospatial software that can work in conjunction or complement GIS.

During the last 20 years GIS have played an increasingly important role in archaeology. Their capacity to work on a multi-layered and multi-scale spatial frame renders them a very flexible tool to handle all spatially referenced information. Nowadays, they are routinely employed in archaeological research and practice with more researchers applying them to address not only landscape but also on site issues. However, their incorporation into the archaeologist's toolbox is relatively recent. The first GIS software (if they could have been called as such back then) were developed in the 60 s and they were restricted to very basic functions, such as the creation of 3D messes or the extraction of contours. During the 70s the first commercial software was developed by the Environmental Systems Research Institute (ESRI), the most successful vendor of proprietary software today. The first applications, however, of GIS to archaeological research were only later developed under the influence of New Geography by the American pioneers of New Archaeology. The positivist approach of New Archaeology was an ideal framework for GIS. Operations such as the delineation of Thiessen polygons, site catchment areas or the development of predictive models of the location of archaeological sites were simple and straightforward operations for GIS, and therefore, had a prolific application during the late 80's and early 90's.

These first GIS were aimed to work with either vector or raster data but nowadays, most available GIS software packages have evolved to include an increasing number of functions that have been usually regarded the realm of other types of software (Wheatley and Gillings, 2002, p. 9). Nowadays it is quite common using GIS as a CAD program, to develop 3D reconstructions and virtual environments, to perform digital

Hector Orengo: University of Sheffield, Sheffield, UK 
image analysis operations and remote sensing analyses, database management and query, and spatial statistics.

Archaeological research is one of the most active fields in the application of GIS today. This is perhaps a consequence of the often-subtle nature of archaeological evidence. Archaeologists are concerned with human behaviour in the past and, it has to be admitted, the traces left by it are mostly elusive and difficult to discern. While most GIS users are concerned with the physical environment as it is today, landscape archaeologists (the group most involved in the use of GIS) have to employ a much wider variety of GIS-based techniques to be able to discover, analyse and compare traces of past human activities. It is therefore common in archaeological research to integrate GIS with remote sensing and other geospatial techniques. Another limiting factor is that, in many instances, current environmental datasets can rarely be applied to solve archaeological questions since large-scale landscape modifications during the last centuries could have been important and these need to be taken into account when studying ancient environments. Also, the interest of the landscape archaeologist lies in the study of societies in the past, in how they interacted with their environment and in the consequences of these interactions. This is a particularly difficult topic to explore since it does not only take into account environmental variables but also social, cultural, temporal and economic inputs for which information is often lacking. Research in archaeology and the humanities also incorporates subjective and imprecise data that can be difficult to map (for a more thorough discussion see Jessop 2008). In order to explore all these aspects archaeologists use most techniques available in the GIS toolbox, such as topographic analyses, including several types of visibility analysis, and least cost route modelling; predictive site location modelling, hydrographic analysis, network analysis, and so on.

Archaeologists focussing on site-oriented research or excavations have also stretched GIS resources to their limit due to the very high spatial resolution and exactitude required in the recording of the excavation process, its finds and features. Nowadays archaeological recording procedures include the use of complex spatially aware object-oriented databases, 3D reconstruction techniques, and so on. Perhaps this is further illustrated by the multiple scripts developed specifically for archaeological purposes or the adaptation of open source GIS software to archaeological research.

The number of techniques currently put into archaeological enquiry is abundant and chances are that a single project will require the use of many of those. However, it should be noted that no single GIS includes all possible functionalities. As in most other types of software the selection of the software package is an essential one and some familiarity with GIS is needed in order to choose the most appropriate software suite for each research question/project. Nevertheless, most GIS are modular in nature, that is, they are composed of a collection of modules directed to do specific functions. The majority of GIS can easily increase their capabilities by integrating new modules (also called extensions, scripts, plugins or add-ons) to add specific function- 
alities. As GIS users become aware of the particular functionalities and modules available in different packages, it is more common to employ several software packages, performing specific tasks or analyses using the most adequate or convenient module to the task at hand.

\subsection{What is Open Source Software?}

Open source software (OSS) is any computer software the source code of which is made available through a license according to which the copyright holder provides the right to download, analyse, modify and distribute the software to anyone and for any purpose (including commercial). "Free and open source software" (FOSS) is another commonly employed term that includes both open source and free software. As stated by the Free Software Foundation, the term "free" refers to freedom more than to "gratis" although, FOSS, at least as described by the Open Source Initiative and the Free Software Foundation, is by definition no-cost. Indeed, as stated by the Free Software Foundation:

\footnotetext{
"The term "open source" software is used by some people to mean more or less the same category as free software. It is not exactly the same class of software: they accept some licenses that we consider too restrictive, and there are free software licenses they have not accepted. However, the differences in extension of the category are small: nearly all free software is open source, and nearly all open source software is free."
}

Also, there is plenty of closed source software (often proprietary) distributed at no cost through freeware, shareware or other types of licenses. That must be kept in mind since, as it will be indicated in later sections, this causes strong restrictions in the development of an online community of contributors and the long-term improvement and maintenance of the software. Proprietary software is also commonly referred to as commercial although, as Ducke has stated (2012, p. 572), this might be a somewhat misleading term because most open source software is developed through hired programmers and therefore involves commercial processes.

In this chapter the terms OSS and FOSS will be used interchangeably. All the software discussed in the text will refer to open source unless stated otherwise.

\subsection{Why Use Open Source GIS?}

There are some evident advantages in the use of open source GIS for archaeological research and indeed much literature has been dedicated to it (see, for example, Serlorenzi 2013). One of these is the transparency of open source software where the source code can be studied and changed. Although most archaeologists do not have programming skills and therefore cannot take direct advantage of this feature, the 
availability of source code makes it open to public scrutiny and improvement (Chambers, 2008, p. 6-7) and, as a consequence, most (or at least those widespread) open source GIS software is today very reliable and rapidly evolving. As pointed out by several authors (e.g. Hafer and Kirkpatrick 2009, p. 126; Ducke 2012; Ince et al. 2012), open source software should be in fact a requirement in research since the principles of scientific enquiry demand research to be open to scrutiny. Proprietary software's inaccessible source code, not being open to public scrutiny and reproducibility, prevents understanding of the processes involved in the production of outputs and thus their critical evaluation. As Morin et al. have stated (2012, p. 159), close source programmes act as 'black boxes', effectively hindering good scientific practice.

Another very important advantage of open source software, and one with many consequences (see below), is that it is distributed at no cost and it is publicly available. The very high price of proprietary GIS software often prohibits its acquisition by small enterprises or research centres, which would be obliged to spend tens of thousands of pounds for a single license (not including extensions) that needs to be renewed constantly. Neither archaeological research nor commercial archaeological practice can be included in those disciplines with higher returns on investment, and therefore, the use of proprietary geospatial software in archaeology is particularly taxing.

A consequence of the free availability of open source programs is that they are portable, that is, the user can have as many copies installed in as many computers as necessary. In other words, the user does not need to go to a specific workstation in a lab in order to access a GIS-enabled computer. Providing the user has the necessary permissions to install software, open-source software can be installed in any computer. Due to the transferability of open source GIS, having experience in the use of open source GIS programs is an increasingly important factor in the geospatial job market. Open source GIS users are able to move to different working environments and to continue being productive without the need to adapt to the specific software choice available at their new location, since they can always continue using their preferred open source GIS at no additional cost for the employer. This is in line with the tendency towards a growing market for open source GIS users. Enterprises with geospatial analysis needs can save significant amounts of money in adopting FOSS or changing their proprietary software for FOSS and accordingly they might be interested in their personnel to have experience with open source GIS.

Free software not only means a substantial saving of money but also implies the existence of a vibrant online community of users and multiple learning resources, which include tutorials and other learning resources, such as datasets. The most widespread open source GIS like GRASS GIS or QGIS offer plenty of manuals, tutorials and video tutorials, online help and multiple busy forums maintained by thriving communities where the user can learn and discuss applications. Following the open approach to software distribution, manuals and tutorials are available to download for free. This renders FOSS GIS an excellent choice for both the amateur and the experienced user. 
Also, as a consequence of their large online community, the most senior GIS projects have been running for a long time and therefore have evolved into very secure and competent packages. They continuously release updated versions in which 'bugs' or programming errors are solved and new functionalities implemented. As most of these are to some degree dependent on public participation and online communities of users and developers they are also very sensitive to user demands and feedback and usually incorporate improvements and innovations based on these (Brandtzæg et al., 2010).

The public nature of open source GIS has also resulted in the extensive availability of plug-ins that, given the modular nature of GIS, accounts for an important increase in open source GIS functionality with respect to that available in commercial software in which extensions are relatively minimal, usually more complex, and extremely expensive if the user requires several of them. Having access to many different modules is very important since they can save the user significant effort and time. While using proprietary software one is limited to the modules available within the software or those extensions one can buy, most extensions or plugins in open source software are small scripts directed to accomplish a simple function written by other users with the same needs. Software suits such as QGIS offer plugin managers where extensions can be easily queried, downloaded and installed without the need to restart the program. Open source add-ons have another important advantage: many of them are crossplatform. That is, they can be loaded on more than one open-source software, making life easier for those users who perform repetitive tasks in more than one program. This is important since, as any GIS experienced user would agree, it is very difficult to work with a single GIS package. The modular nature of GIS accounts for a large number of available modules with different performances and capabilities. It is therefore important to identify which modules are the most appropriate for the task at hand.

Many open source GIS programs can run in windows, Mac or Linux. This is a substantial difference with commercial software, such as ArcGIS, IDRISI or ERDAS IMAGINE (some of the most widespread geospatial analysis packages) that can only be installed on Microsoft Windows, forcing users of other operating systems to install windows in virtual environments (which can be a source of multiple problems) or in a partition of their hard drive. Many of them like QGIS or GVSIG provide versions that can run on tablets or, even mobiles, turning a portable device into a GPS-integrated mobile GIS, a truly useful feature for such common fieldwork as archaeological survey.

FOSS projects tend to join efforts thanks to the willingness of their developing teams, users and the fact that their modules' source code is publicly available. That is also a substantial difference with proprietary software where competence for market shares would, to say the least, restrict collaborations between competing enterprises. This tendency results in FOSS being developed more rapidly and incorporating more and more advanced functionalities than its commercial counterparts. 


\subsection{Problems with Open Source GIS}

The growing use of open source GIS applications can be easily explained by the several advantages outlined above but it still remains a long way back from that of commercial software (Figure 5.1), particularly from ESRI's ArcGIS, which keeps a large portion of the market shares. This might be due to several reasons. Some of them can be labelled as 'psychological', such as the feeling most users have that paying for something is justified by receiving a higher quality product, and therefore, free software must be of lower quality. Commercial software have also a much longer history of use. Many users find it difficult to adapt to new software packages, while the new versions of their traditional software incorporate similar (if not the same) commands, terminology, graphic interface, etc., which softens the transition process. Related to this issue is the widespread use of proprietary software in the classrooms and GIS laboratories of universities and research centres where most GIS users are trained. Most of this teaching is still based on the repetition of practical processes directed at solving specific case-studies and not on the learning of the concepts, functions, applications and basic structure of GIS, which renders the adaption to new GIS software difficult.

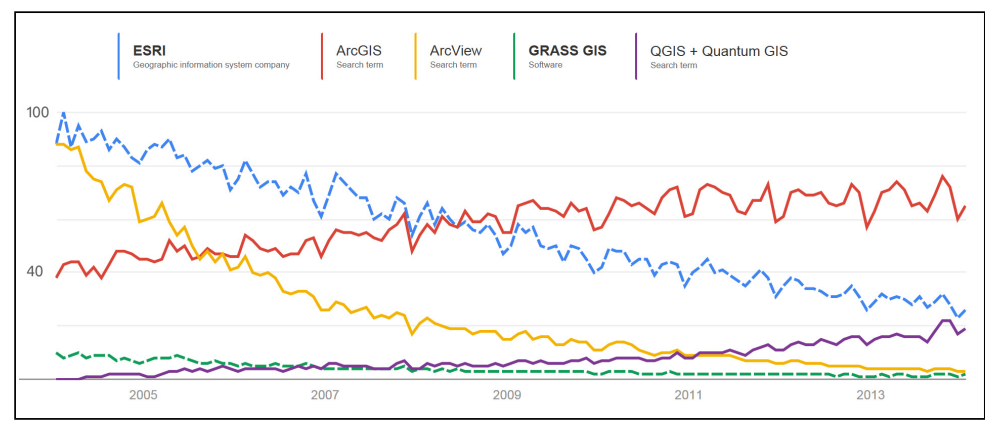

Figure 5.1: Google Trends search comparison between different GIS-related search terms

However, part of this still marginal use of open source GIS can also be related to some intrinsic problems of this type of software:

- While the strong focus of many GIS projects on the development of a community of users and developers is key to their success, it also brings some problems: Outdated scripts as a consequence of the lack of a central organisation arranging the distribution of new releases and updating the extensions, many of which are made by individual programmers which might not be willing to keep updating it for latter releases. However, the efforts made by OSGeo (the Open Source Geospatial Foundation) have made a significant step towards the interoperability and maintenance of geospatial open source code, significantly reducing the risk of outdated modules in the projects it manages. 
- Abandoned open source GIS development projects are also common, mainly in archaeology where projects are linked to relatively short term funding, and thus, long-term maintenance, updating and support are rarely contemplated. However, a tendency towards embedding single projects within larger community-lead projects such as OSGeo can improve this situation (Ducke, 2012). To avoid this, the novice open source GIS user could direct his/her GIS interests towards large, welldeveloped projects, such as the ones described in the section on open source GIS programs.

- Long-term support and the availability or consistency of documentation is also very dependent on the vitality of the online community of users and/or developing organism (Steiniger and Bocher, 2009). As in the previous point this can be easily solved by employing well-established open source packages.

\subsection{Common Misconceptions Regarding Commercial and Open-Source Software}

Many of the misconceptions dealt with here might have been painfully true ten years ago. Many long-term open source users will still remember difficulties in installation, frequent crashing or bugs preventing certain scripts to run properly. These current misconceptions therefore include, as usually said about legends, an element of truth. However, updating and clearing old beliefs is necessary, particularly when dealing with fast developing computer software.

- 'Complex graphic user interfaces (GUIs) or the use of command lines makes difficult the use of open source GIS'. This might be true for some open-source packages, for example GRASS GIS, which although much improved from previous versions, still employs a GUI that can be considered strange for those used to ESRI's products. However, most established open source GIS suits available today offer similar environments to those available in ESRI's software and some of them were, in fact, designed to serve as an easy-to-used graphic interface for more complex packages.

- 'Lack of compatibility with proprietary formats'. Although some open source GIS, such as GRASS, still use their own native format to which imported files need to be transformed before being able to work with them, most open source packages accept proprietary or other common file formats. In fact they employ very sophisticated geospatial libraries, such as GDAL/OGR, that are able to import most file formats. It is also common to find plugins, that allow importing and working with unusual and specific file formats. All in all, the truth is that open source software has a much wider compatibility than commercial software.

- 'Commercial software is of higher quality, more secure and less-prone to crashing'. As with other of the listed misconceptions this might have been true some 
time ago but not anymore. In fact, my own personal experience as a higher education academic who is forced to use both open source and proprietary software is that the former, particularly ArcGIS, has a tendency to crash more often and more destructively. In my experience, this seems to be a pretty common conviction among academics and archaeologists involved in both types of GIS distribution strategies.

- 'Open source GIS is useful for computer geeks or programmers but not adequate for the non-technical GIS user: a lot of the open source GIS software expects the user to develop his/her own modules and tools'. Modern open source GIS is developed with the non-technical user in mind and one of its priorities is promoting the widespread use of open source tools. However, contrary to commercial software suits, open source promotes user-developed innovations and active participation in its development. It is curious that such a promotion might be considered in detriment to its usability since it is this openness to public design that renders open source GIS such a complete and advanced set of tools.

- 'Open source software is geared towards the Linux community'. Similar to the previous point, this idea arises from the first open source program available, GRASS, which was developed in UNIX/Linux and until its version 6.4 in 2010 there was not a native Windows version available (although previous versions could be run on Windows using Cygwin). Still today the Windows native GRASS has reduced functionality compared to that distributed for Linux. Nowadays GRASS and other open source software packages, such as gvSIG and QGIS (which were multi-platform since their first releases), are distributed for all major operating systems including Mac OS. This is something than many non-Windows users are demanding from their proprietary GIS software developers/vendors for many years now.

- 'Commercial software offers more help and support'. Related to this idea, is the conception that it is difficult to learn how to use open source GIS. For the most established open source suits, such as GRASS (Neteler \& Mitasova 2008), QGIS (Thiede et al., 2013; Graser, 2013) and gvSIG (Arnalich and Ton-That, 2010), there are available manuals that can be bought at any major book seller or online bookstore and are as expensive as any handbook dedicated to commercial software. These also offer extensive documentation, help, online forums and, as commented before, an active online community always prone to help with doubts and problems. As in the case of commercial software there are plenty of enterprises that offer training and support services for open source software (Steiniger and Bocher, 2009). QGIS, for example, has listed in its website 27 international organisations that offer commercial training, support and programming for QGIS, GRASS and other open source software. Learning how to use open source GIS should not be expected to be easier or cheaper than learning how to use commercial software.

- 'Proprietary software offer long-term support'. While, as mentioned previously, it is true that many open source GIS projects, particularly those specifically directed 
to archaeological purposes (Ducke, 2012), never had continuity after their first development, the largest open source GIS projects are running for decades and they can be considered very effective in offering long-term support. It is, in fact, commercial software the one posing real risks to its long-term use. Many will still remember with yearning all those old scripts written in Avenue lost when migrating from ArcView to ArcGIS. Some others will still keep their old ArcView so they can use these scripts that incorporated functionalities that ArcGIS still does not have or requires an expensive extension to perform. Although the non-technical open source GIS user can also lose the scripts by updating their software, any user with programming skills can update a script or contract a company to do so thanks to the public availability of source code (Steiniger and Bocher, 2009). This would not be possible with close source software. More importantly, proprietary software licenses pose specific problems for long-term use. Steiniger and Bocher (2009, p. 1364) clearly illustrate this with several examples:

"Other problems may occur due to vendor license changes or changes of license distribution rules. For example, the developers of Forestry GIS were hit by the first case; subsequently, as of November 2005 they were not allowed to freely distribute updates of their software because the license terms for the used proprietary GIS kernel were changed (ForestPal, 2008). The second case happened to Oxford Archaeology, an educational charity, which faced problems when ESRI Inc. was announcing to change their criteria for the awarding of educational licenses (see Cook 2008)."

- 'Commercial software is technically more advanced'. Just having a look to the list of modules and operations offered by GRASS or SAGA it becomes transparent that this does not hold true. Many of the newer open source projects offer a limited quantity of operations, but, the strong tendency that open source software has to join resources (and modules) in open repositories that are accessible to open source desktop GIS programs, promises great analytical possibilities even to the smallest project. Several authors (Camara et al., 2000; Steiniger and Bocher, 2009) have already commented the slow implementation rate of innovations in proprietary software, many of which have been available on open source GIS for years. Could it possibly be that this deceleration in innovation is due to a conscious effort to sell specific extensions or updates at a conveniently paced rate for market purposes?

Several of those misconceptions, such as complex GUIs and lack of compatibility with common file formats, seem to have their origins in older versions of GRASS as this has been the forefront and most visible representative of open source GIS software, but it is now time to re-evaluate them and acknowledge the huge progress made by FOSS during the last years. 


\subsection{Which Open-Source Desktop GIS is more Convenient?}

Although many remote packages exist, this paper focuses on desktop applications that are software installed and run on personal computers, are not executed on a server and are not remotely accessed or controlled from or by a different computer. The following list is based on the author's experience, expertise and research interests and it is therefore not exhaustive, the reader is, therefore, directed to more specialised publications for comparison between different software choices (Sherman, 2008; Steiniger and Bocher, 2009; Hengl et al., 2009; Cattari and Clutterbuck, 2011; Steiniger and Hunter, 2013) and is encouraged to try and experience other packages to find the one that better adapt to his/her experience and needs.

\subsubsection{GRASS (Geographic Resources Analysis Support System) GIS}

This is the big name in open source GIS, with thirty years since its original development by the U.S. Army Corps of Engineers - Construction Engineering Research Laboratories (USA-CERL). GRASS was developed as a raster-analysis program and this is perhaps why its raster analysis capacities are its main analytical feature but its latest versions confidently support vector creation editing and analysis, database management systems, network analysis, LiDAR data processing, image processing and analysis (including multispectral data analysis), spatial modelling with capacity to deal with multi-temporal data, 3D raster (voxel) development, analysis and advanced visualization and graphics, and maps production. The last release of GRASS contains over 400 modules (Neteler et al., 2012) that can be increased with more than 200 userdeveloped extensions, some of them created specifically for archaeological applications that can be found in the OSGeo AddOns webpage.

GRASS offers an excellent option for advanced GIS users and it has commonly been applied to archaeological research. Some of its capabilities are unique in both commercial and open source desktop GIS. It is the only GIS desktop software that allows creating and analysing 3D surfaces or voxels, and NVIZ, its 3D visualization engine (Figure 5.2), allows single and multi-map 2D perspective support but also multidimensional visualisation, voxel visualisation as isosurfaces or cutting plane rendering, animations, full resolution exports, and 3D queries (Hofierka et al., 2002; Neteler et al., 2012). Apart from these fancy tools, GRASS can import almost all types of existing geospatial file types, which makes it extremely useful if only to load, see and export one's files in other formats. Although still experimental, two new features in the latest stable release (GRASS 6.4.3) provide important new functionalities. The new graphical modeller offers a tool for the implementation of complex or repetitive workflows, saving a substantial amount of time to the user. The graphic composer solves some of the issues with GRASS map composition and graphic export capacities.

GRASS can also be linked to other open source software, such as QGIS and the statisti- 


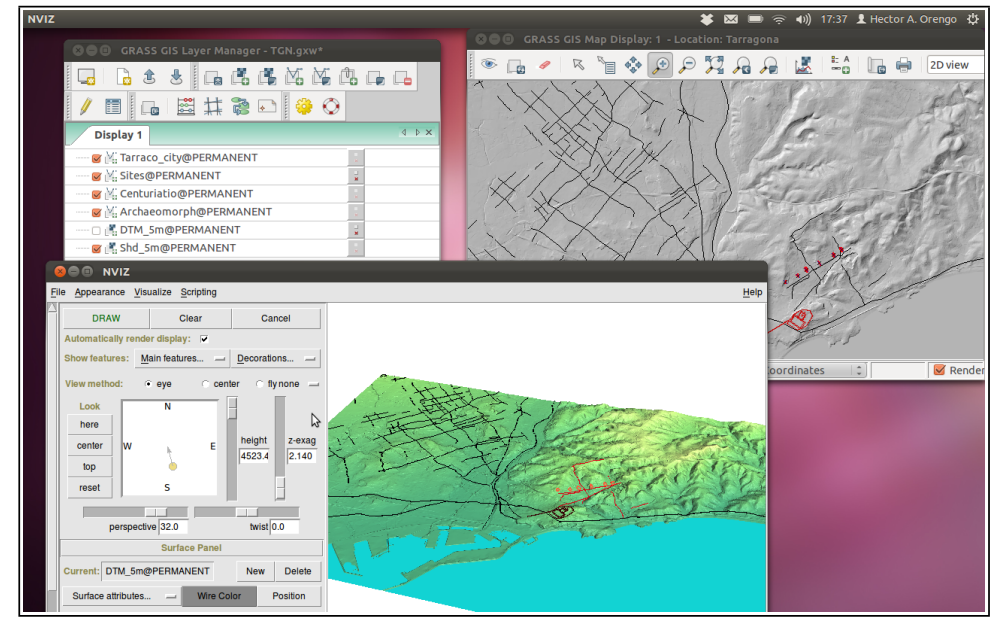

Figure 5.2: GRASS GIS running on Linux Ubuntu showing the Layer Manager, Map Display and the NVIZ module

cal package R, to increase and/or complement its capabilities. In archaeology GRASS has been employed in analysis of past landscapes (e.g. Barton, Ullah and Mitasova 2010; Barton, Ullah and Bergin 2010; Ullah 2011), predictive modelling of routes and archaeological sites (Indruszewski and Barton, 2006; Orengo and Miró, 2011), visibility analyses (Lake et al., 1998; Cooper, 2010), for the volumetric reconstruction of the excavation process (Lieberwirth, 2008; Orengo, 2013), and many other archaeologyrelated tasks.

GRASS GIS has, however, some problems that the unexperienced user should take into account when firstly approaching it. GRASS's 'floating window' graphical user interface (Figure 5.2), although much improved from earlier versions, can be a bit awkward to users accustomed to ESRI products. It is also difficult to start experimenting with GRASS functionalities without a basic knowledge of GIS and geographical concepts: for the program to start running you need to specify your project location, projection and coordinate reference system. Also before you can start using or even visualising your data you need to import it into GRASS's own native format. In this regard, its quality and coherence can be one of the problems hindering the widespread adoption of GRASS. Equally, the complexity of the analyses performed by some of the modules available in GRASS makes some of them difficult to apply without a good knowledge of the principles or theory on which these were based. 


\subsubsection{QGIS (Formerly Known as Quantum GIS)}

QGIS started developing in 2002 as a response to the expert-oriented design of GRASS. It aimed to act as a data viewer and, at a later stage, provided a graphical user interface for GRASS, increasing in this way its analytical capacities. Since then, however, QGIS has become the popular open source desktop GIS choice (see the increase in QGISrelated web searches during the last years in figure 5.1). This might be due to its smooth and clear design(Figure 5.3), its ease of use and its restricted needs in terms of disc space, RAM and processing power. Its simple approach to the editing of vector features contrasts with most other commercial and open source suits. Similarly to many other open source packages it is cross-platform, running on Linux, Mac OSX, Windows and Android.

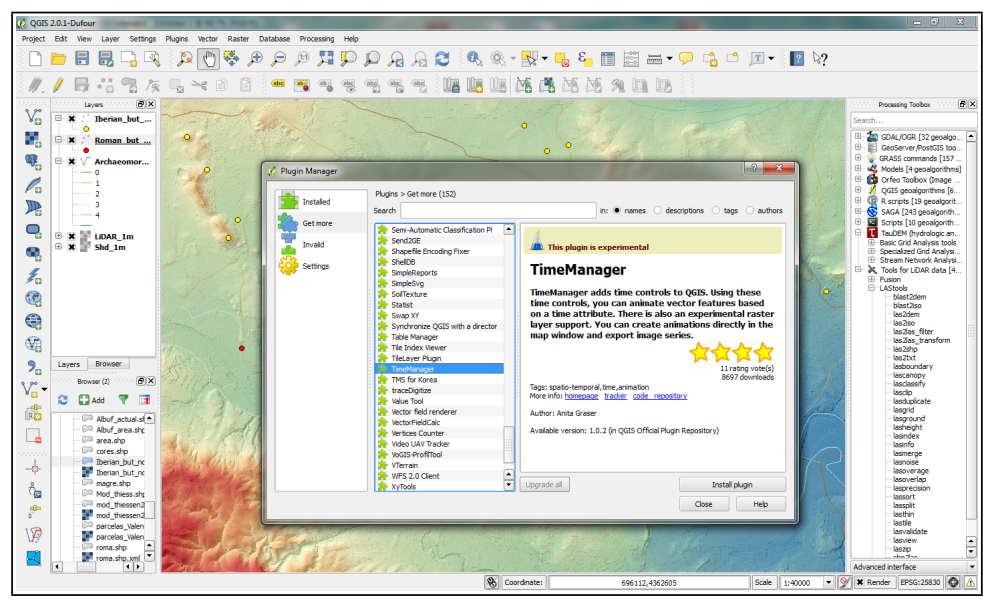

Figure 5.3: QGIS running natively on Windows 7 showing its attractive GUI with the Processing Toolbox (at the right side) and the Plugin Manager (in the centre)

The latest version of QGIS (Dufour 2.0.1) provides access through its toolbox (Figure 5.3) to modules not only developed for GRASS but also for Sextante (a series of raster tools initially developed for gvSIG), SAGA GIS, Orfeo Toolbox, R package for statistical computing, etc. However, not all modules from the original software are accessible through the QGIS toolbox. For instance, only 157 modules of GRASS modules and 243 from the almost 600 available in SAGA can be accessed. A great advantage of using the last QGIS version is that GRASS modules run from QGIS will not require the files to be transformed into the GRASS native format. QGIS plugin manager (Figure 5.3) provides a really simple way to query and install extensions without the need to exit the program. The 174 extensions available, some of them preloaded in the program, 
include all kinds of vector and raster analysis, including simple network capabilities and multispectral image analysis.

QGIS can load and edit ESRI's shapefiles and other common vector formats without the need to transform them. It can also load and work with the most common raster file types. Its many extensions provide compatibility with not so common geospatial file formats. Also, QGIS allows on-the-fly coordinate reference system transformation, which enormously simplifies the creation of projects joining data in different projections or coordinate systems, saving the need to transform the files for their integration.

Just as GRASS does, QGIS incorporates a graphical model builder to simplify and automate workflows and a print composer, which allows a smooth production of attractive maps.

Therefore, QGIS is a very complete desktop GIS with important capabilities for visualisation, analysis and cartographic production. Its ease of use and complete documentation, help files, manuals and on-line communities make QGIS the obvious choice for those starting to use open source GIS.

\subsubsection{SAGA (System for Automated Geoscientific Analyses) GIS}

SAGA GIS has been under development since 2001 and in 2004 its source code was publicly released. The latest release (2.1.0) runs under Windows and Linux and contains 586 modules distributed in 67 libraries, which renders it one of the most complete GIS packages in the market. It is a raster-oriented GIS but it has capacity to work with and edit vector data although, compared with the other packages described here, these are complex and hard to use. Its 3D render engine is efficient and fast but limited in the range of file types it can represent. SAGA is not a good choice for GIS starters or even archaeologists working with vector data. Its graphic export capabilities are very limited and the GUI (Figure 5.4) still has some bugs that limit its use. However, SAGA GIS is very conceptual in its development and users with a good knowledge of rasterbased GIS concepts will feel comfortable with it. It was designed for a simple and effective implementation of spatial algorithms. Its set of tools is particularly efficient for Digital Terrain Models analysis, hydrologic analysis and environmental modelling and incorporates multiple tools for multispectral image analysis.

\subsection{4 gvSIG (Generalitat Valenciana Sistema d’Informació Geogràfica)}

This open access GIS project is unusual for being developed by a public administration. In a few years since it was publicly released it has become well known and a number of archaeologists employ it for their daily GIS work. It is developed in Java, which makes it flexible and easy to adapt to different operative systems. Its amiable graphic interface (Figure 5.5), ease of use (its vector editing tools are very simple and 


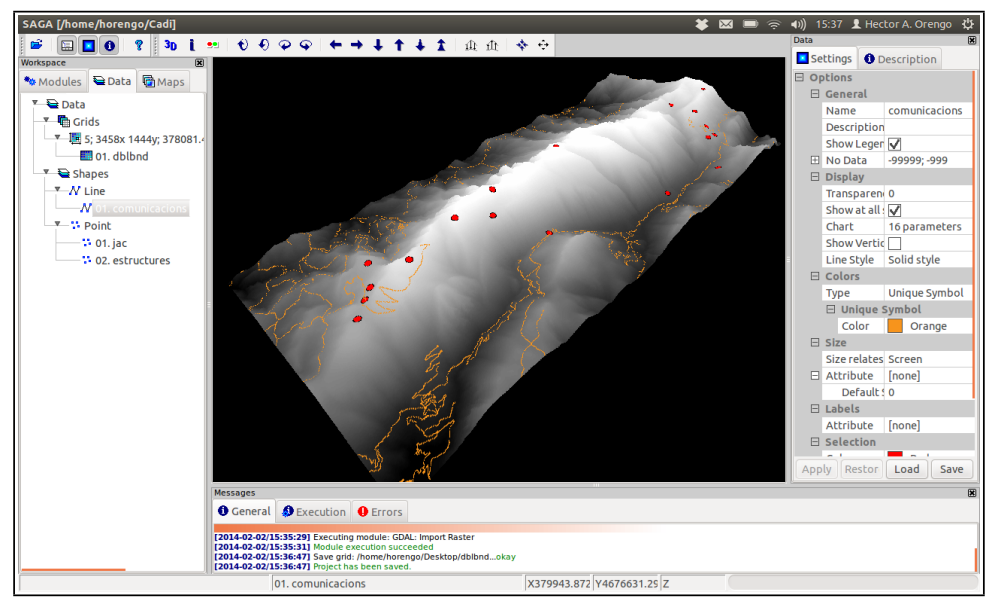

Figure 5.4: SAGA GIS running on Linux Ubuntu with the 3D window integrating raster and vector data

efficient) together with its capacity to work natively with the most common file types, justify its expanding use during the last few years. Several add-ons, such as Sextante, incorporate advanced analytical capabilities to work with raster data. Other useful extensions include 3D analysis, network analysis, remote sensing and LiDAR data analysis, although this last is still restricted in its capacities. It should be noted, however, that most of these extensions are still not available for the latest stable version (2.0) and some of these may not ever be. GvSIG add-ons Manager (Figure 5.5) provides a simple and useful way to manage and get new plugins.

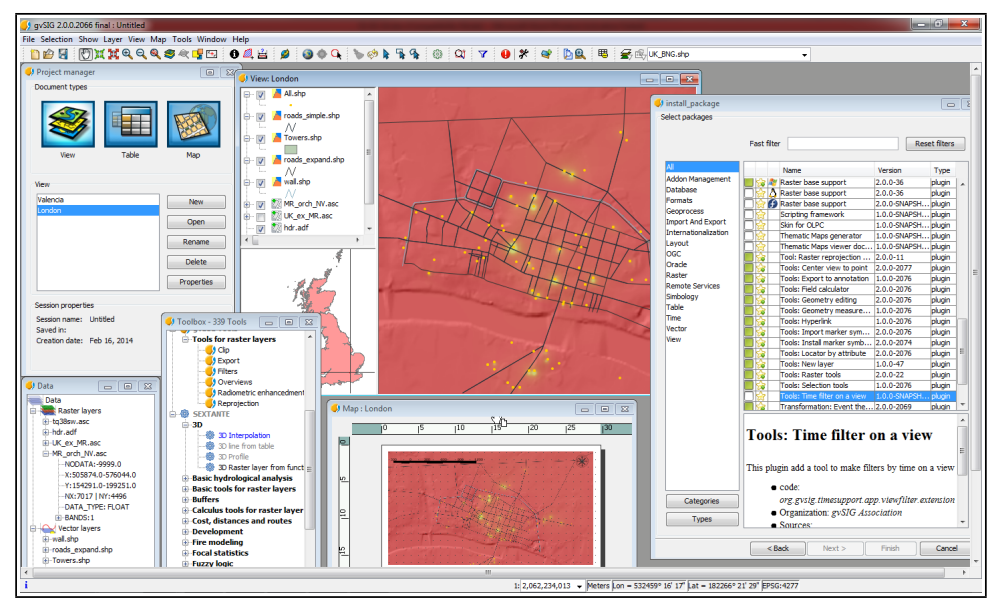

Figure 5.5: gvSIG running on Windows 7 displaying the Project Manager (top left), the View Window (top centre), the Map Window (bottom centre), and the Add-ons Manager (right) 
This, therefore, is an excellent software package for both starters and advanced users. Its current desktop stable version is very secure and the existence of a mobile option makes it particularly useful for those working in the field. gvSIG is currently in the OSGeo's “incubation” process, that is, is being evaluated to be one of OSGeo's projects. Being part of OSGeo will ensure or at least facilitate the long-term maintenance and the growth of resources available for the communities of users and developers of gvSIG.

It is interesting to note, in regard to the applicability of gvSIG to archaeologyrelated tasks, that Oxford Archaeology has developed a version of this software: gvSIG OA Digital Edition. gvSIG OADE is fully portable, has a simpler menu and installation process and incorporates GRASS and Sextante modules. However, gvSIG OADE is based on gvSIG v.1.10 and does not include the latest updates and improvements present in gvSIG 2.0.

\subsubsection{Other Open Source Geospatial Software}

GIS is rarely used in isolation and, consequently, it is worth briefly reviewing other geospatial applications that can be used in conjunction with GIS to solve all the archaeologist's geospatial needs. Some FOSS packages, although providing some GIS capabilities, are oriented towards specific types of analysis. Programmes such as the public domain HEC-RAS and the open source Calypso, provide advance hydrologic modelling, which include flood analysis. Some other software packages that can also be used in combination with GIS are more directed towards Remote Sensing, such as ILWIS, the BEAM Toolbox (with VISAT GUI), Orfeo Toolbox and Opticks (which is currently being considered as an OSGeo project).

Other remote sensing-related group of programs are those directed to the treatment of LiDAR data. Although some of the open source GIS software explored before offer the possibility of importing, treating and transforming LiDAR data, only a few packages, such as rapidlasso's LAStools (note that not all tools are open source but all are free for non-commercial use) and MeshLab, offer the possibility to deal with LiDAR-generated point clouds without the need to import them into specific GIS software.

Another category of software able to generate georeferenced point cloud data is the photogrammetric 3D reconstruction programs. During the last few years several open source projects have been aimed at the development of software capable of automated image processing for 3D reconstruction. Most of these, being relatively recent, are still difficult to use with few manuals or online help and, when present, unattractive GUIs. However, some of these provide excellent photogrammetric capabilities, able to match their most common commercial counterparts (Remondino et al., 2012). Perhaps the best known among these is Bundler (Snavely et al., 2008), which is now somewhat outdated and complex to use for those starting with photogrammetric 3D modelling. VisualSfM, free for personal, non-profit, or academic use, was developed 
by Changchang $\mathrm{Wu}$ and offers a similar solution to that of Bundler but with a much easier to follow workflow and a simple GUI. Finally, Apero and MicMac, a set of programs developed by the Matis laboratory of the French IGN, provide advanced and accurate capabilities for the production of photogrammetric 3D models. An advantage of MicMac over other packages is that it can be used to produce georeferenced orthoimages, without the need to employ a GIS program. MicMac is distributed under the French CeCILL-B license (fully compatible with BSD-like licenses).

Both photogrammetric and LiDAR-derived point clouds can be loaded, treated and exported with open source programs, such as Meshlab or ParaView, which, with the PCL plugin, allow users to access algorithms from the Point Cloud Processing Library (PCL). ParaView also offers other 3D advanced visualisation capabilities and is able to load and explore different types of volumetric data.

\subsection{Open Geospatial Data?}

After having reviewed some of the different open source GIS options available to the archaeologist it is necessary to consider the availability of geospatial data needed to perform GIS analysis. This is a critical issue since without data it is not possible to perform any GIS analysis (Cattari and Clutterbuck, 2011, p. 24). Although most of the previously described packages offer datasets for training purposes, these are very restricted in spatial terms and will not provide data on a project's specific area of research. Of course the archaeologist is expected to be able to generate his/her own data from field survey, excavation, site and heritage records, document and map analysis, and so on. Archaeological geospatial data, however, are difficult to come by since, as Jessop has pointed out (2008, p. 44), 'the capture of spatial data is a major task in many projects and thus the scale of effort and cost of creating digital datasets make some groups reluctant to share them without charge'. Institutions such as English Heritage and initiatives such as the Portable Antiquities Scheme offer free access to their spatially enabled databases after subscription. However, for those working on a regional scale or in landscape projects, data such as digital terrain models, geological maps or aerial imagery are necessary and few projects' budget can afford the resources necessary to generate them.

International efforts like the INSPIRE Directive, which aims to produce an EU spatial data infrastructure to facilitate public access and share environmental spatial information across Europe, are incomplete and institutions like the Ordnance Survey in England or the Institut Géographique National in France still charge very expensive prices for digital geographic data. Some other geographic data providers, such as Edina Digimap and Mimas Landmap for the UK's higher education and academic research community, charge expensive subscription rates than can only be afforded by big enterprises.

Fortunately, following the philosophy developed in the European INSPIRE Direc- 
tive, more countries, such as Spain, provide vast amounts of reliable geographical information at no cost following a registration process. Although the availability of open access geographical data is highly variable depending on the country some international bodies such as the European Environment Agency, which provides environmental data for Europe, deliver quality open access data in common GIS formats with good associated metadata, that is, information on how the information was gathered and the data treated and produced.

The US NASA and USGS provide worldwide satellite imagery and elevation datasets, in many cases free of charge upon registration through USGS EarthExplorer, USGS GloVis, Global Data Explorer. The European Space Agency also provides access to satellite imagery following registration and, depending on the data sought, a lengthy process involving the setting up of a project. Global elevation datasets can also be found at CGIAR-CSI GeoPortal (SRTM data at $90 \mathrm{~m} /$ cell resolution) and at the ASTER GDEM site (at 30m/cell resolution).

There are also repositories of free digital geospatial data, often contributed by a community that can be downloaded freely from the Internet. Nonetheless, many of these datasets do not usually incorporate metadata that would allow the user to have a good idea of the processes that were followed to create, transform and distribute them. This is an essential requirement for geospatial information and the use of these data may be risky when highly accurate information is required.

\subsection{Conclusions}

The advantages of employing open source GIS for archaeological research and practice are evident and have been thoroughly described here. Open source GIS software have undergone dramatic changes during the last few years and are now complete and secure packages that can successfully address all of the archaeologist's needs.

The coming years will probably see an increase in the capacities, efficiency and ease of use of these programmes, with more resources being available for training and specialised use. This will be, in part, a consequence of the development of foundations and associations, such as OSGeo, which support the collaborative development, maintenance and collaboration of open source geospatial software. This is very significant since the nurturing of cross-project collaboration and integration of open source resources generated by multiple projects will, no doubt, produce the most advanced and secure geospatial software available. This tendency toward the integration of open source projects is already a reality in GIS suits like QGIS through extensions and plugins. An increase in the integration of capabilities in open source GIS it is thus expected to materialise in the next few years that will, hopefully, result in complete geospatial information systems.

The innovation generated by joined open source initiatives may also force com- 
mercial software to increase the capacity and quality of their products and hopefully reduce their prices.

\section{Bibliography}

Arnalich, S. and Ton-That, T. (2010), gvSIG y Cooperación: CÃşmo construir e incorporar un Sistema de Informaciẽşn GeogrÃafica a tu proyecto, Arnalich.

Barton, C. M., Ullah, I. I. and Bergin, S. (2010), 'Land use, water and mediterranean landscapes: modelling long-term dynamics of complex socio-ecological systems', Philosophical Transactions of the Royal Society A: Mathematical, Physical and Engineering Sciences 368(1931), 5275-5297.

Barton, C. M., Ullah, I. and Mitasova, H. (2010), 'Computational modeling and neolithic socioecological dynamics: a case study from southwest asia', American Antiquity 75(2), 364-386.

Brandtzæg, P. B., Følstad, A., Obrist, M., Geerts, D. and Berg, R. (2010), Innovation in online communities-towards community-centric design, in 'User centric media', Springer, pp. 50-57.

Camara, G., Souza, R., Pedrosa, B., Vinhas, L., Monteiro, A. M. V., Paiva, J., Carvalho, M. d. and Gattass, M. (2000), Terralib: technology in support of gis innovation, in 'll Workshop Brasileiro de Geoinformática, Geolnfo2000', Vol. 2, pp. 1-8.

Cattari, G. and Clutterbuck, R. (2011), 'Free open-source geographical information systems for irish archaeology', Archaeology Ireland pp. 21-25.

Chambers, J. (2008), Software for data analysis: programming with $R$, Springer.

Cook, J. (2008), 'Dear ESRI, it's not me, it's you', Open Source Computing and GIS in the UK. URL: http://www.archaeogeek.com/blog/2008/03/12/dear-esri-its-not-me-its-you/

Cooper, J. (2010), 'Modelling mobility and exchange in pre-columbian Cuba: GIS led approaches to identifying pathways and reconstructing journeys from the archaeological record', Journal of Caribbean Archaeology pp. 122-137.

Ducke, B. (2012), 'Natives of a connected world: free and open source software in archaeology', World Archaeology 44(4), 571-579.

ForestPal (2008), 'Forestry GIS'.

URL: http://www.forestpal.com/fgis.html

Graser, A. (2013), Learning QGIS 2.0, Packt Publishing Ltd.

Hafer, L. and Kirkpatrick, A. E. (2009), 'Assessing open source software as a scholarly contribution', Communications of the ACM 52(12), 126-129.

Hengl, T., Grohmann, C., Bivand, R., Conrad, O. and Lobo, A. (2009), 'SAGA vs GRASS: a comparative analysis of the two open source desktop GIS for the automated analysis of elevation data', Proceedings of Geomorphometry .

Hofierka, J., Parajka, J., Mitasova, H. and Mitas, L. (2002), 'Multivariate interpolation of precipitation using regularized spline with tension', Transactions in GIS 6(2), 135-150.

Ince, D. C., Hatton, L. and Graham-Cumming, J. (2012), 'The case for open computer programs', Nature 482(7386), 485-488.

Indruszewski, G. and Barton, C. (2006), Simulating sea surfaces for modeling viking age seafaring in the baltic sea, in 'Digital discovery: exploring new frontiers in human heritage. CAA 2006. Computer applications and quantitative methods in archaeology. Proceedings of the 34th conference, Fargo, United States, April 2006', pp. 616-30.

Jessop, M. (2008), 'The inhibition of geographical information in digital humanities scholarship', Literary and Linguistic Computing 23(1), 39-50.

Lake, M., Woodman, P. and Mithen, S. (1998), 'Tailoring GIS software for archaeological applications: an example concerning viewshed analysis', Journal of Archaeological Science 25(1), 27-38. 
Lieberwirth, U. (2008), 'Voxel-based 3D GIS: Modelling and Analysis of Archaeological Stratigraphy', Beyond Illustration: 2D and 3D Digital Technologies as Tools for Discovery in Archaeology. Oxford: Archaeopress pp. 85-94.

Morin, A., Urban, J., Adams, P., Foster, I., Sali, A., Baker, D. and Sliz, P. (2012), 'Shining light into black boxes', Science 336(6078), 159-160.

Neteler, M., Bowman, M. H., Landa, M. and Metz, M. (2012), 'GRASS GIS: A multi-purpose open source GIS', Environmental Modelling \& Software 31, 124-130.

Orengo, H. A. (2013), 'Combining terrestrial stereophotogrammetry, DGPS and GIS-based 3D voxel modelling in the volumetric recording of archaeological features', ISPRS Journal of Photogrammetry and Remote Sensing 76, 49-55.

Orengo, H. A. and Miró, C. (2011), Following Roman waterways from a computer screen: GISbased approaches to the analysis of Barcino's aqueducts, in P. Verhagen, A. Posluschny and A. Danielisová, eds, 'Go Your Own Least Cost Path: Spatial Technology and Archaeological Interpretation: Proceedings of the GIS Session at EAA 2009, Riva Del Garda', British Archaeological Reports, International Series. Oxford, Archaeopress, pp. 47-53.

Remondino, F., Del Pizzo, S., Kersten, T. P. and Troisi, S. (2012), Low-cost and open-source solutions for automated image orientation-a critical overview, in 'Progress in Cultural Heritage Preservation', Springer, pp. 40-54.

Serlorenzi, M., ed. (2013), ARCHEOFOSS Free, Libre and Open Source Software e Open Format nei processi di ricerca archeologica. Atti del VII Workshop (Roma, 11-13 giugno 2012).

Sherman, G. (2008), Desktop GIS: Mapping the Planet with Open Source Tools, Pragmatic Bookshelf.

Snavely, N., Seitz, S. M. and Szeliski, R. (2008), 'Modeling the world from internet photo collections', International Journal of Computer Vision 80(2), 189-210.

Steiniger, S. and Bocher, E. (2009), 'An overview on current free and open source desktop GIS developments', International Journal of Geographical Information Science 23(10), 1345-1370.

Steiniger, S. and Hunter, A. J. (2013), 'The 2012 free and open source GIS software map-a guide to facilitate research, development, and adoption', Computers, Environment and Urban Systems 39, 136-150.

Thiede, R., Sutton, T., Düster, H. and Sutton, M. (2013), The Quantum GIS Training Manual: A Comprehensive Introduction to Quantum GIS, Locate Press.

Ullah, I. I. (2011), 'A GIS method for assessing the zone of human-environmental impact around archaeological sites: a test case from the Late Neolithic of Wadi Ziqlâb, Jordan', Journal of Archaeological Science 38(3), 623-632.

Wheatley, D. and Gillings, M. (2002), Spatial Technology and Archaeology, London, Taylor \& Francis. 